\title{
Adipocyte-derived exosomal miRNAs: a novel mechanism for obesity-related disease
}

\author{
Sarah C. Ferrante', Evan P. Nadler ${ }^{1,2,3}$, Dinesh K. Pillai ${ }^{1,4,5}$, Monica J. Hubal',2, Zuyi Wang ${ }^{1}$, Justin M. Wang', \\ Heather Gordish-Dressman' ${ }^{1}$ Emily Koeck ${ }^{2}$, Samantha Sevilla ${ }^{2}$, Andrew A. Wiles ${ }^{1}$ and Robert J. Freishtat ${ }^{1,4,6}$
}

BACKGROUND: Obesity is frequently complicated by comorbid conditions, yet how excess adipose contributes is poorly understood. Although adipocytes in obese individuals induce systemic inflammation via secreted cytokines, another potential mediator has recently been identified (i.e., adipocytederived exosomes). We hypothesized that adipocyte-derived exosomes contain mediators capable of activating end-organ inflammatory and fibrotic signaling pathways.

METHODS: We developed techniques to quantify and characterize exosomes shed by adipocytes from seven obese (age: $12-17.5$ y, BMl: $33-50 \mathrm{~kg} / \mathrm{m}^{2}$ ) and five lean (age: $11-19$ y, BMl: $\left.22-25 \mathrm{~kg} / \mathrm{m}^{2}\right)$ subjects.

RESULTS: Abundant exosomal miRNAs, but no mRNAs, were detected. Comparison of obese vs. lean visceral adipose donors detected 55 differentially expressed miRNAs $(P<0.05$; fold change $\geq|1.2|)$. qRT-PCR confirmed downregulation of miR$148 \mathrm{~b}$ (ratio $=0.2(95 \%$ confidence interval $=0.1,0.6)$ ) and miR$4269(0.3(0.1,0.8))$, and upregulation of miR-23b $(6.2(2.2,17.8))$ and miR-4429 (3.8 (1.1-13.4)). Pathways analysis identified TGF- $\beta$ signaling and $\mathrm{Wnt} / \beta$-catenin signaling among the top canonical pathways expected to be altered with visceral adiposity based on projected mRNA targets for the 55 differentially expressed miRNAs. A select mRNA target was validated in vitro. CONCLUSION: These data show that visceral adipocytes shed exosomal-mediators predicted to regulate key end-organ inflammatory and fibrotic signaling pathways.

O besity is a systemic inflammatory state (1) associated with chronic inflammatory and metabolic diseases impacting nearly every organ system within the body (2-4). Previous studies showed that visceral adipocytes promote systemic inflammation by secreting mediators such as interleukin (IL)6 , tumor necrosis factor (TNF)- $\alpha$, and leptin, among others (5). These mediators are secreted into the circulation $(5,6)$ and affect distant organs increasing the risk of type 2 diabetes, atherosclerosis, cardiovascular disease, asthma (4), and several forms of cancer. In addition, obesity is known to increase systemic TGF- $\beta 1$ levels, which activate fibroblasts to differentiate into myofibroblasts, increasing the release of extracellular matrix proteins (7). Extracellular matrix protein accumulation can disrupt physiological organ architecture, resulting in fibrotic disease such as that seen in nonalcoholic fatty liver disease (NAFLD) $(8,9)$.

Recently, the exosome has garnered attention as an important mediator of intercellular communication. Exosomes are actively shed endocytic vesicles that contain and transport functional mRNAs, miRNAs, and proteins between cells $(10,11)$. In obese individuals, adipocyte-derived exosomes are known to contribute to the development of insulin resistance via activation of adipose-resident macrophages and secretion of proinflammatory cytokines that can result in insulin resistance (12). However, exosomes may also carry proinflammatory factors via the circulation (13) and interact with remote cell types to promote inflammation through activation of contributory pathways $(14,15)$. We hypothesize that visceral obese adipocytes shed exosomes that contain mediators capable of activating end-organ inflammatory and fibrotic signaling pathways.

Herein, we isolated, quantified, and characterized exosomes from surgically acquired human visceral and subcutaneous adipose tissue from obese and lean adolescents. We isolated total exosomal RNA detecting only miRNA but not mRNA. Therefore, we profiled exosomal miRNAs focusing on obese vs. lean visceral adipose expression due to the association between accumulating visceral adipose and increased cardiometabolic disease risk $(5,16)$. miRNA profiles show downregulation of several miRNAs in obese compared to lean visceral adipocyte exosomes that target TGF- $\beta$ and $W n t / \beta$-catenin signaling, which would be expected to activate these pathways in endorgans by decreasing negative regulation of gene expression. This finding provides important insight into a direct mechanism by which obesity may mediate comorbid disease.

\section{RESULTS}

\section{Subject Demographics}

Obese $(n=7)$ and lean $(n=5)$ adolescents were enrolled on the day of surgery. The mean age at the time of surgery was $15.0 \pm 0.7 \mathrm{y}( \pm$ SEM $)$ for obese participants and $15.6 \pm 1.4 \mathrm{y}$ for

\footnotetext{
'Department of Integrative Systems Biology, Children's National Medical Center, Washington, DC; ${ }^{2}$ Sheikh Zayed Institute for Pediatric Surgical Innovation, Children's National Medical Center, Washington, DC; ${ }^{3}$ Division of Pediatric Surgery, Children's National Medical Center, Washington, DC; ${ }^{4}$ Department of Pediatrics, George Washington University School of Medicine and Health Sciences, Washington, DC; ${ }^{5}$ Division of Pulmonary and Sleep Medicine, Children's National Medical Center, Washington, DC; ${ }^{6}$ Division of Emergency Medicine Children's National Medical Center, Washington, DC. Correspondence: Robert J. Freishtat (rfreishtat@childrensnational.org) 
lean participants $(P=0.70)$. Obese participants had a presurgery mean BMI of $39.8 \pm 2.0 \mathrm{~kg} / \mathrm{m}^{2}$ and for lean participants the mean BMI was $23.2 \pm 0.5 \mathrm{~kg} / \mathrm{m}^{2}(P<0.001)$. All participants

Table 1. Demographics of patients enrolled in study

\begin{tabular}{cccl}
\hline Age (years) & Sex & BMI & \multicolumn{1}{c}{ Race } \\
\hline Lean & & & Hispanic \\
11 & F & 22 & Hispanic \\
13 & F & 22 & White \\
17 & F & 24 & African American \\
18 & F & 23 & African American \\
19 & F & 25 & \\
Obese & & & African American \\
12 & F & 49 & Hispanic \\
13 & F & 33 & White \\
14 & F & 40 & African American \\
15.5 & F & 39 & African American \\
16 & F & 43 & Hispanic \\
17 & F & 35 & African American \\
17.5 & F & 50 & \\
\hline
\end{tabular}

were female and from diverse racial backgrounds. Selected clinical characteristics are shown in Table 1.

\section{Macrophage Activation State in Obese and Lean Adipose}

Adipose macrophages have different activation states; proinflammatory (known as M1 and classically activated by interferon- $\gamma$ or lipopolysaccharide) or anti-inflammatory (known as M2 and alternatively activated by IL-13 or IL-4) (17). We characterized the activation state of infiltrating macrophages in the adipose from all participants by immunohistochemistry for $\mathrm{M} 1$ (i.e., $\mathrm{CD} 40^{+}$, a macrophage surface receptor involved in proinflammatory pathways) $(17,18)$ and $\mathrm{M} 2\left(\mathrm{CD} 163^{+}\right.$or $\left.\mathrm{CD} 206^{+}\right)$activation states (Figure 1a). Obese visceral adipose shows a higher number of $\mathrm{CD}_{4} 0^{+}$macrophages than lean visceral adipose (lean $_{\text {subcutaneous }}$ (mean \pm $\mathrm{SEM})=1.3 \pm 0.5$ events per field (range $=0.0-3.3)$, lean $_{\text {visceral }}=$ $1.3 \pm 0.4(0.7-2.3)$, obese subcutaneous $_{\text {s. }}=3.3 \pm 0.6(0.0-3.3)$, obese $_{\text {vis }}$ ceral $=5.0 \pm 0.8(2.7-8.0)$; comparison of lean visceral $_{\text {vs. obese }}$ visceral $P=0.028$ ) (Figure $1 \mathbf{b}$ ). In addition, obese subcutaneous adipose showed a higher number of $\mathrm{CD} 163^{+}$macrophages than lean subcutaneous adipose (obese subcutaneous $=1.0 \pm 0.3(0.3-2.0)$ vs. lean $\left.n_{\text {subcutaneous }}=0.2 \pm 0.1(0.0-0.3) ; P=0.04\right)$. No differences

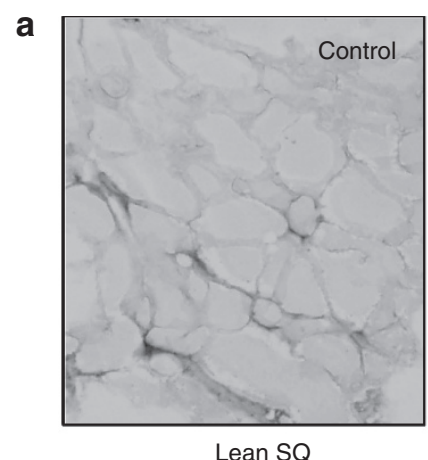

Lean $S Q$

b

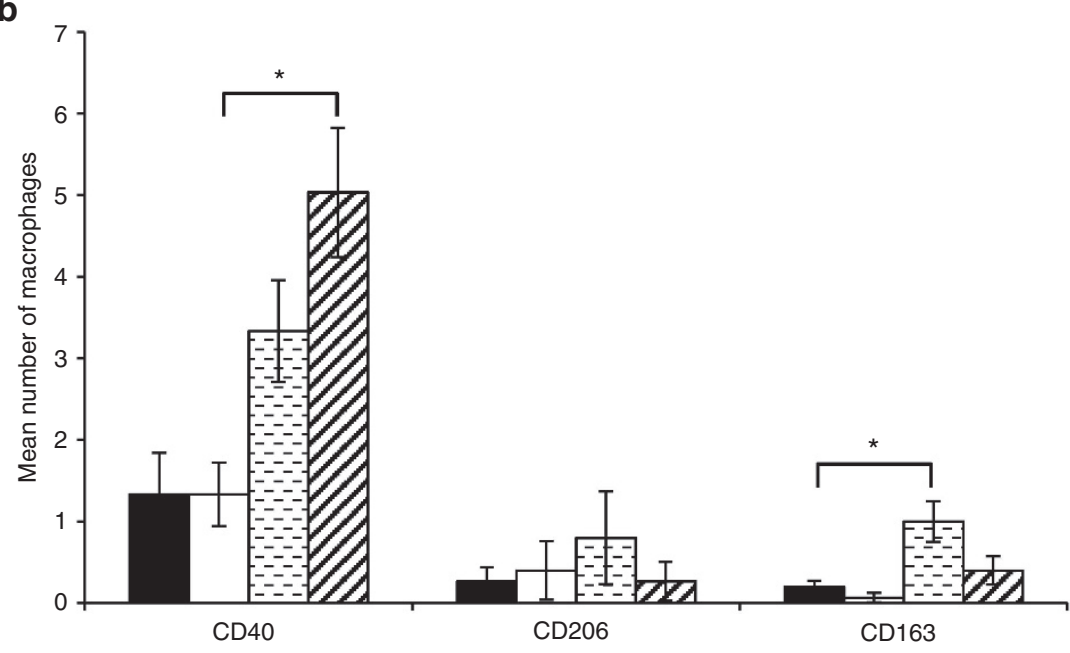

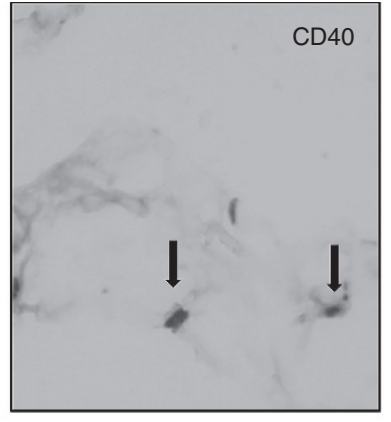

Obese visceral

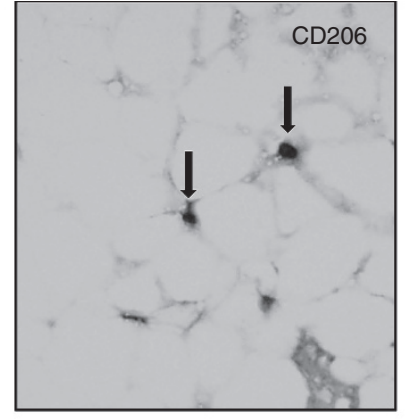

Obese SQ

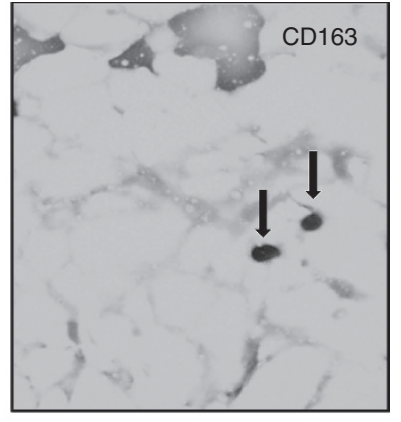

Obese visceral

C

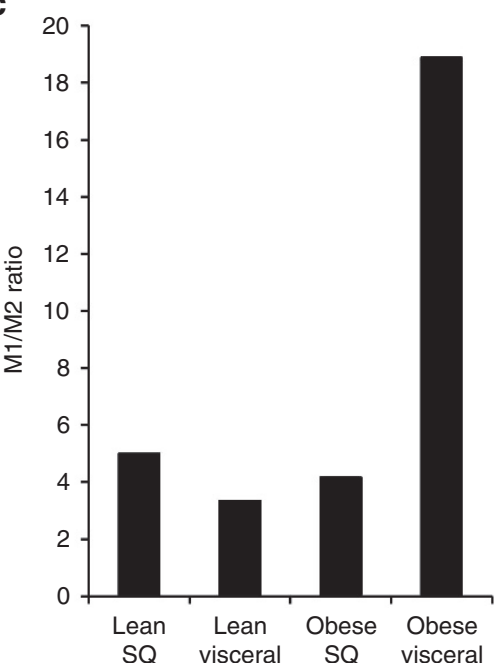

Figure 1. M1:M2 Macrophage immunohistochemistry of adipose tissues. (a) Frozen sections of adipose from visceral and subcutaneous (SQ) depots were stained for CD40 (M1), CD206 (M2), and CD163 (M2), in seven obese and five lean individuals. Representative samples are shown at 20× magnification. (b) Obese visceral adipose shows a higher number of CD40+ macrophages than lean visceral adipose. In addition, obese subcutaneous adipose has a higher number of $\mathrm{CD}_{163^{+}}$macrophages than lean subcutaneous adipose. No significant differences were found for $C D 206^{+}$macrophages. ${ }^{*} P<0.05$ determined by a two-sided Student's $t$-test; error bars represent \pm SEM; key: Lean SQ (black square), Lean visceral (white square), Obese SQ (dotted square), and Obese visceral (lined square). (c) The obese visceral adipose depot showed the highest M1:M2 ratio compared to every other condition. 
were found for CD206 ${ }^{+}$macrophages. Consistent with previous studies $(17,19)$, the obese visceral adipose depot showed the highest M1:M2 ratio compared to every other condition $\left(\mathrm{M} 1: \mathrm{M} 2\right.$ ratios: Obese $_{\text {visceral }}=18.9$, obese subcutaneous $=4.2$ lean $_{\text {subcu- }}$ ${ }_{\text {taneous }}=5.0$, lean $\left._{\text {visceral }}=3.3\right)($ Figure $1 \mathrm{c})$.

\section{Sizing and Quantification of Adipocyte-Derived Exosomes}

We verified size for isolated adipocyte-derived exosomes using nanoparticle tracking analysis that demonstrated all vesicles were within the 50-100 nm size range. To quantify the isolated exosomes, we adaptedbead-based flowcytometryapproaches. Wefirst attached PKH67 prelabeled exosomes to latex beads via binding to the CD63 antigen, a known exosomal marker (20-22). Figure 2 a depicts the principle of this technique: With increasing concentration of exosomes in a suspension, more fluorescently labeled exosomes are bound to beads, therefore the fluorescence increases proportionally. Using the same labeling technique on known concentrations of commercially available exosome standards,
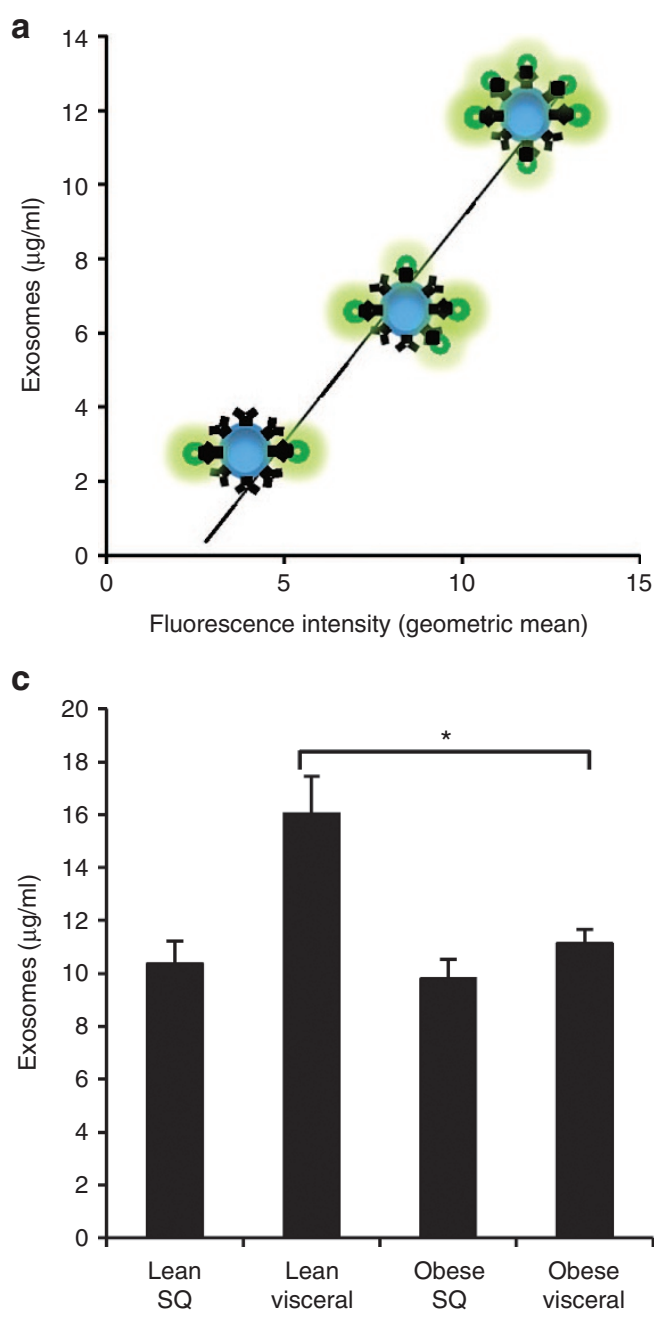

we generated a reproducible standard curve of geometric mean fluorescence intensity vs. concentration ( $\mu \mathrm{g} / \mathrm{ml})$ (Figure $2 \mathrm{~b}$ ). The derived concentrations of exosomes isolated from lean and obese adipose are shown in Figure $2 \mathrm{c}$ (lean $_{\text {subcutaneous }}=$ $10.4 \pm 0.8 \mu \mathrm{g} / \mathrm{ml}$, lean visceral $=16.0 \pm 1.4 \mu \mathrm{g} / \mathrm{ml}$, obese subcutaneous $=$ $9.8 \pm 0.7 \mu \mathrm{g} / \mathrm{ml}$, and obese $\left._{\text {visceral }}=11.1 \pm 0.6 \mu \mathrm{g} / \mathrm{ml}\right)$. Only lean visceral adipocyte-derived exosomes are more concentrated than obese visceral adipocyte-derived exosomes $(P \leq 0.03)$. In addition, as the BMI of the donor increases, visceral adipocytederived exosome concentration decreases, $\mathrm{R}^{2}=0.46$ (Figure $2 \mathrm{~d}$.

\section{Characterization of Adipocyte-Derived Exosomes}

Given variable resident macrophage infiltration of the adipose, it was important to verify the cellular origin of the isolated exosomes. Consequently, we chose three markers: Fatty acid binding protein 4 (FABP4), an adipocyte differentiation marker (23); DLK/Pref-1, a preadipocyte marker (24); and CD14, a macrophage marker (25). Lean adipocyte exosomes

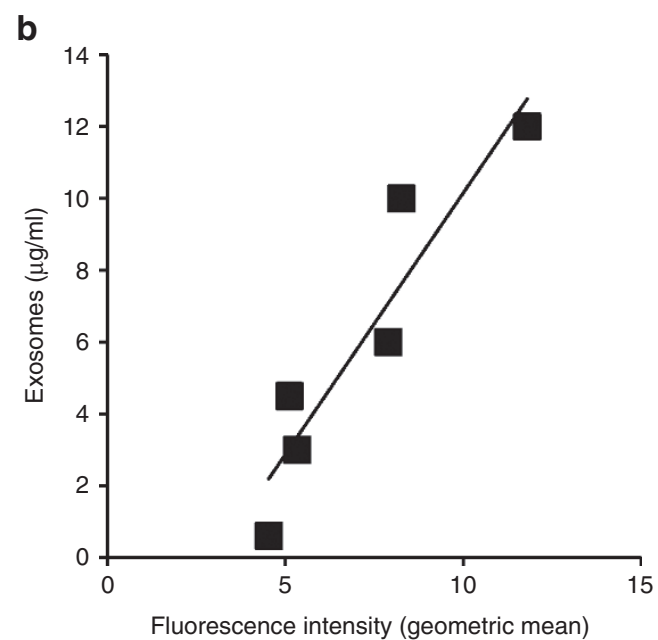

d

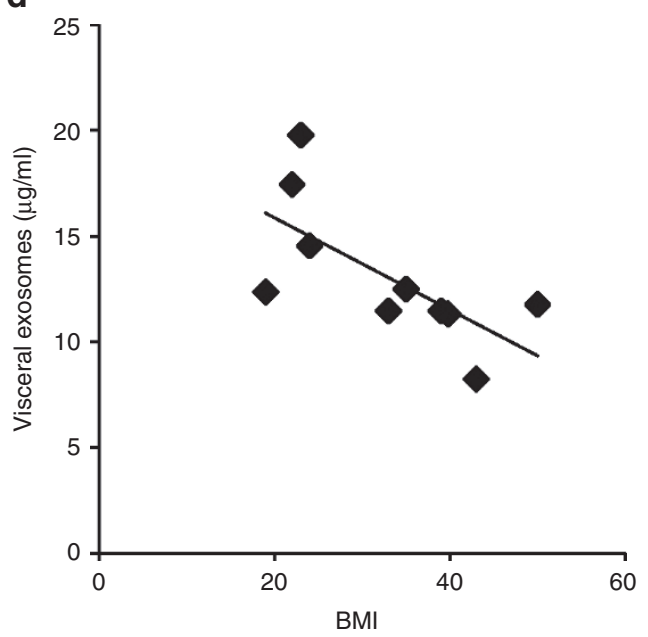

Figure 2. Quantification of adipocyte-derived exosomes using bead-based flow cytometry. (a) Depiction of the bead-based flow cytometry technique: Increasing exosomal concentration results in increased number of exosomes attached to beads, therefore proportionally increasing geometric mean fluorescence intensity of PKH67 staining. (b) Generated standard curve and coefficient of determination of commercially available exosomal standards $\left(R^{2}=0.87\right)$. (c) Concentration of isolated exosomes from a standardized starting amount of obese $(n=7)$ and lean $(n=5)$ adipose. Lean visceral adipocytederived exosomes are significantly more concentrated than those from obese visceral adipose. ${ }^{*} P<0.05$ determined by a two-sided Student's $t$-test; error bars represent \pm SEM. (d) As BMI increases, visceral adipocyte-derived exosome concentration decreases $\left(R^{2}=0.46\right)$. 


\section{Articles | Ferrante et al.}

were almost entirely $\mathrm{FABP}^{+}(99.7 \pm 0.001 \%)$. In addition, many beads bound DLK/Pref- $1^{+}$exosomes $(43.4 \pm 0.014 \%)$. Importantly, very few exosomes were $\mathrm{CD}^{+} 4^{+}(2.8 \pm 0.004 \%)$. Similarly, obese adipocyte exosomes were also almost all $\mathrm{FABP}^{+}(99.8 \pm 0.001 \%)$ and CD14- $(96.7 \pm 0.003 \%)$ with a similar percentage of beads binding DLK/Pref- $1^{+}(47.5 \pm 0.027 \%)$. We found no differences between conditions (Figure 3 ). The endothelial marker CD31 was not detected.

\section{Differential Expression of miRNA in Obese and Lean Visceral Exosomes}

We could detect no mRNA in isolated exosomes, before or after amplification, by either microfluidic methods or qRT-PCR for the ubiquitous housekeeping gene GAPDH. However, we did detect abundant miRNA by qRT-PCR for the endogenous control, u6snRNA. Amplified exosomal RNA was verified using a $1.5 \%$ agarose gel electrophoresis (Supplementary Figure S1 online). Statistical analyses of miRNA microarray expression revealed that 88 mature miRNAs are present at significantly different levels in exosomes from obese visceral compared with lean visceral adipose (Supplementary Table S1 online). Ingenuity Pathways Analysis software (Ingenuity, Redwood City, CA) mapped the 88 differentially expressed mature miRNAs to 55 known miRNAs based on current miRNA annotations. We found that these 55 differentially expressed mature miRNAs putatively target 7,789 mRNAs. Among the top ranked canonical pathways (Supplementary Table S2 online) represented by these mRNAs are TGF- $\beta$ signaling $\left(P=8.3 \times 10^{-}\right.$ ${ }^{10}$, number of predicted molecules targeted $=54$ out of 89$)$ and Wnt $/ \beta$-catenin signaling $\left(P=4.6 \times 10^{-10}, 93\right.$ out of 171 predicted molecules targeted) (Figure 4 ). In particular, miR -23b, miR140-3p, miR-148b, and miR-182 are experimentally validated to target these pathways (26) (annotated on Figure 4a).

Using qRT-PCR, we attempted to confirm the expression differences between obese and lean visceral exosomes for 7 miRNAs (i.e., miR-23b, miR-148b, miR-182, miR-3681, miR4269, miR-4319, and miR-4429) that had the highest number of experimentally validated and/or predicted target mRNAs (i.e., $\geq 3$ ) in the TGF- $\beta$ and $\mathrm{Wnt} / \beta$-catenin signaling pathways. We confirmed significant downregulation of miR-148b (ratio

a
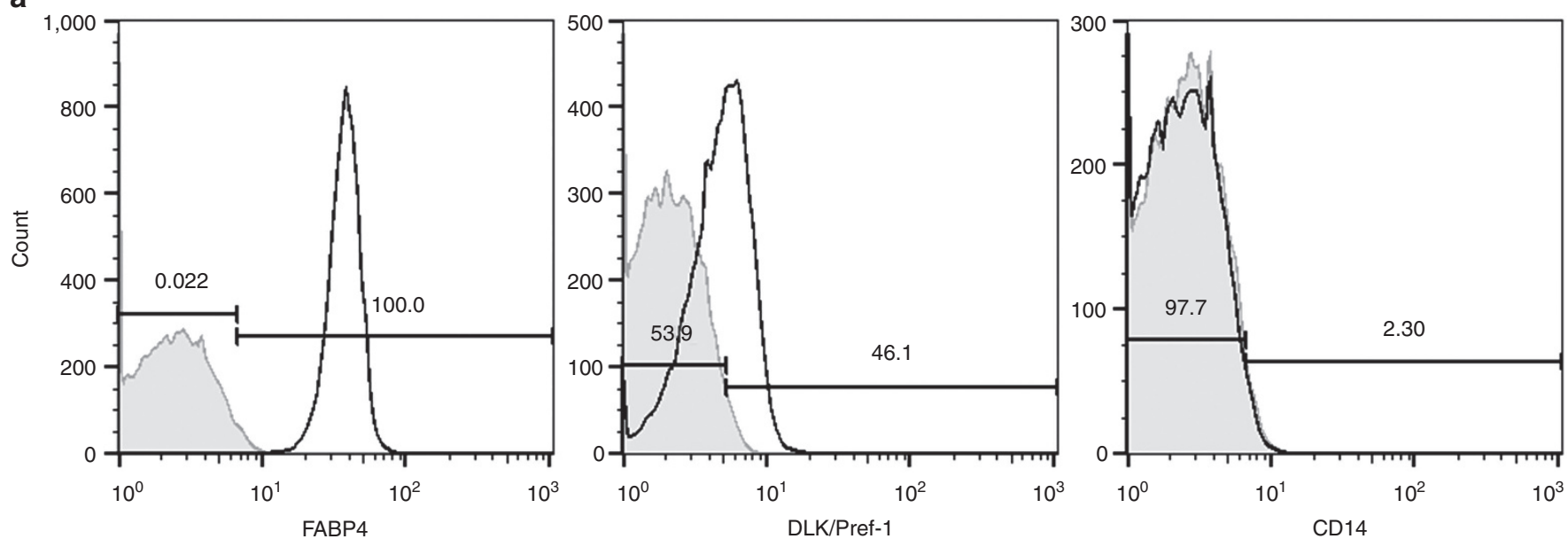

b

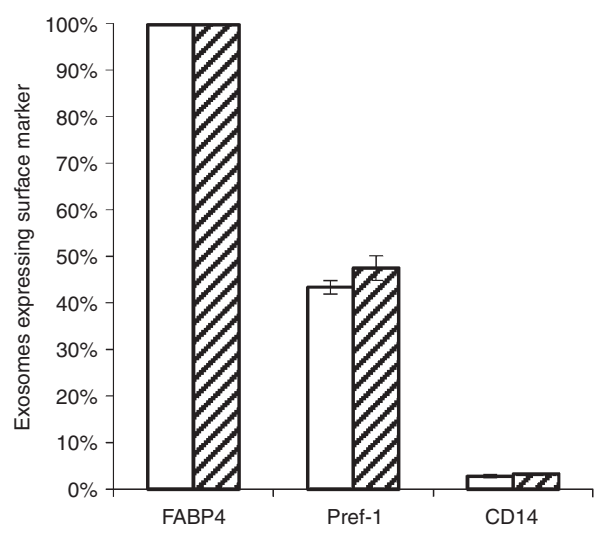

Figure 3. Flow cytometry characterization of adipocyte-derived exosomes. Isolated exosomes from both visceral and subcutaneous depots were characterized for FABP4, Pref-1, and CD14. (a) Representative flow cytometry for FABP4, Pref-1, and CD14 in adipocyte-derived exosomes from a lean subcutaneous sample. (b) Lean adipocyte exosomes $(n=5)$ were almost all FABP4 ${ }^{+}$. In addition, many beads bound DLK/Pref- $1^{+}$exosomes. Very few beads bound $\mathrm{CD} 14^{+}$exosomes. Obese adipocyte exosomes $(n=7)$ were also almost all FABP4 ${ }^{+}$with a similar percentage of beads binding DLK/Pref- $1^{+}$and/or CD14 ${ }^{+}$ exosomes. No differences in marker expression were found between conditions determined by a two-sided Student's $t$-test; error bars represent $\pm S E M$; key: Lean (empty square), Obese (lined square). 
a

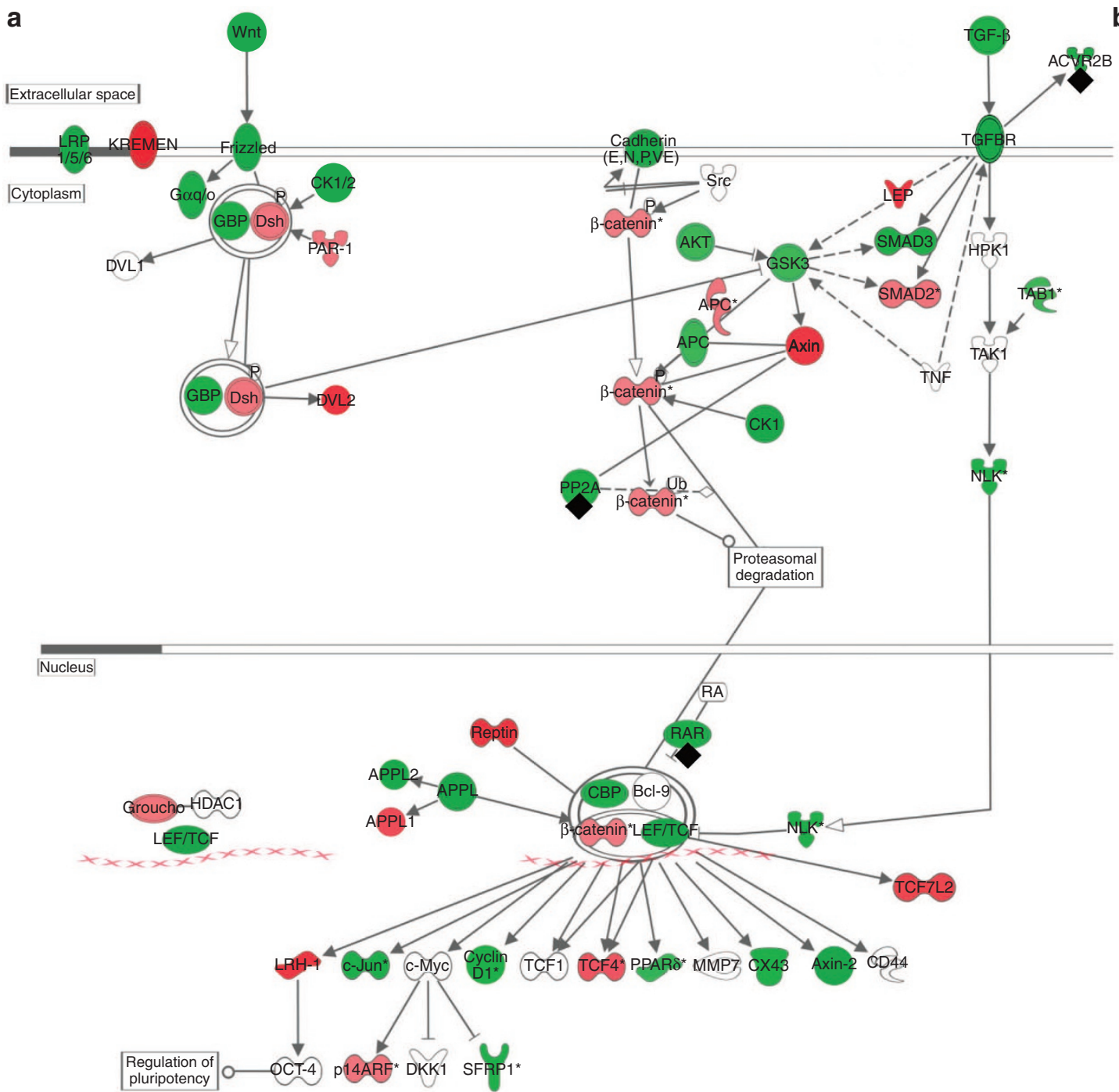

b

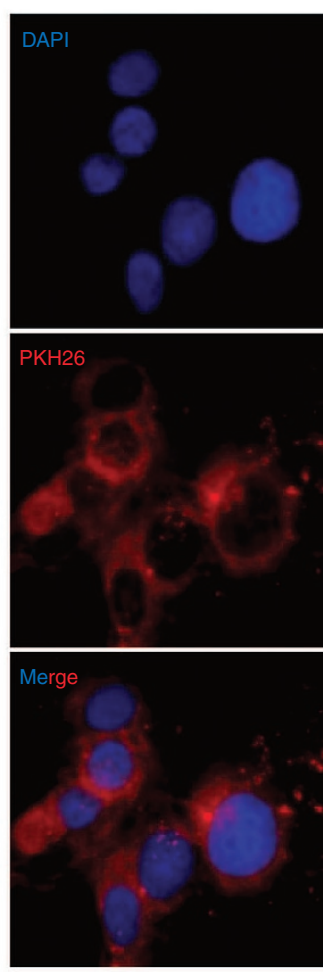

Figure 4. Combined Wnt/ $\beta$-catenin and TGF- $\beta$ signaling pathways and validation. (a) The two canonical pathways were among the top ranked as determined by Ingenuity Pathways Analysis software (Ingenuity, Redwood City, CA). Green indicates predicted downregulation of target transcripts by differentially expressed miRNAs, and red indicates predicted upregulation. Among the altered miRNAs, miR -23b, miR-140-3p, miR-148b, and miR-182 are experimentally validated (annotated with diamond) to target members of the combined pathway. *Genes targeted by multiple miRNAs. (b) Adipocyte exosomal membranes were labeled with PKH26 (red) and coincubated with A549 cells (nuclei stained blue with DAPI) for $24 \mathrm{~h}$. Intracellular PKH26 demonstrates exosomal uptake into A549 cells.

$=0.2(95 \%$ confidence interval $=0.1,0.6))$ and miR-4269 $(0.3$ $(0.1,0.8))$. In addition, we confirmed upregulation of miR-23b $(6.2(2.2,17.8))$ and miR-4429 $(3.8(1.1,13.4))$.

\section{Obese Visceral Adipocyte Exosome Uptake Into Lung Epithelial Cells}

In order to demonstrate that adipocyte-derived exosomes can influence gene expression in cells, we first showed incorporation of these exosomes into cells in vitro. Adipocyte exosome uptake into A549 cells was demonstrated after $24 \mathrm{~h}$ of coculture using PKH26 labeling of obese visceral exosomal membranes (Figure 4b). Immunofluorescent imaging demonstrates that exosome membranes become abundant within the cytoplasm of these cells.

\section{Obese Visceral Adipocyte Exosomes Modify Gene Expression in A549 Cells}

After establishing cellular uptake of adipocyte-derived exosomes, we sought to demonstrate that exosomes could alter gene expression after $24 \mathrm{~h}$ of exposure. We focused this proofof-principle effort on expression of the activin receptor type2B (ACVR2B) in A549 cells. ACRV2B was selected due to its importance in TGF- $\beta$ signaling (27) and the fact that it is targeted by both confirmed upregulated miRNAs in our experiments: miR-23b and miR-4429. Consistent with these miRNA results, ACVR2B was significantly downregulated in the presence of obese compared to lean visceral exosomes (fold change $\left._{\text {obese vs. lean }}=0.19(0.16,0.23)\right)$. 


\section{Articles $\mid$ Ferranteet al.}

\section{DISCUSSION}

As endocytic vesicles capable of transporting functional mRNAs, miRNAs, and proteins between cells over large distances, exosomes are a logical putative contributor to mechanisms by which adipocytes can influence other organ systems. We used novel techniques to quantify and characterize exosomes shed by surgically acquired visceral and subcutaneous adipose from lean and obese individuals. Our exosomal content analyses showed abundant miRNAs. Due to the importance of the visceral adipose depot in obesity-related comorbidities, such as cardiovascular disease and metabolic syndrome (16), we prioritized visceral obese vs. lean comparisons. We found 55 mature miRNAs (with 7,789 confirmed or putative mRNA targets) differentially expressed between these conditions. TGF- $\beta$ and Wnt/ $\beta$-catenin signaling emerged as top canonical pathways targeted by these miRNAs, four of which were confirmed by qRT-PCR: miR-23b, miR-148b, miR-4269, and miR4429. Exosomal uptake by cells was confirmed in vitro along with validation of the predicted downregulation of ACVR2B in the TGF- $\beta$ signaling pathway.

The interaction between Wnt $/ \beta$-catenin and TGF- $\beta$ signaling appears to be important in the development and progression of chronic inflammation and fibrotic disease $(8,28)$. TGF- $\beta$ signaling is implicated in the appearance of extracellular matrix-secreting myofibroblasts $(29,30)$. A recent study demonstrated that canonical Wnt signaling is necessary for TGF- $\beta$ mediated fibrosis (8). Importantly, fibrotic disease can affect multiple organ systems in patients with obesity (9). Furthermore, fat mass extension in humans is correlated with collagen deposition and fibrosis within adipose tissue, leading to systemic metabolic disturbances $(31,32)$.

One aspect of exosome secretion addressed in our study was whether isolated exosomes were of adipocyte or macrophage origin. Previous groups demonstrated that the M1 macrophage activation state is increased in obese visceral adipose and results in the release of mediators that contribute to systemic inflammation (5) and induce insulin resistance (33). Our findings are similar to these previous studies in that visceral adipose from obese participants has higher infiltrating macrophages with $\mathrm{M} 1\left(\mathrm{CD} 40^{+}\right)$activation states. This raised the possibility that macrophage exosomes would contaminate our exosome isolation. However, we confirmed that isolated exosomes express FABP4 and not the macrophage marker CD14. The expression of FABP4 is confined almost exclusively to adipose and adipogenic cell lines, and is highly regulated during adipocyte differentiation (23).

Our finding that lean visceral adipose sheds a higher concentration of exosomes than obese visceral adipose may appear counterintuitive. On the contrary, this is actually an expected finding given that lean visceral adipose has a higher number of adipocytes and more membrane surface area per unit volume resulting in greater exosome shedding. However, in an obese individual, the overall adipose volume is much greater than in lean individuals and thus the relative amount of shed adipocyte exosomes would also likely be greater. This implies that systemic circulation of adipocyte-derived exosomes would be greater in obese individuals, thus magnifying their effect on end-organ mRNA expression.

Because we profiled the contents of isolated exosomes from human adipose ex vivo, the predicted effects of adipocytederived exosomal miRNA on the TGF- $\beta$ and Wnt $/ \beta$-catenin signaling pathways are speculative. However, our data showing exosomal uptake by A549 cells in vitro along with validation of ACVR2B expression differences support this. The downregulation of ACVR2B is very interesting in that activin signaling through this receptor functions similarly to $\mathrm{Wnt} / \beta$-catenin signaling to prevent adipogenesis (34).

To fully understand the function of adipocyte exosomal miRNAs in vivo, it must be determined whether they are shed from adipose into the circulation, if they are extracted by cells in end organs, and the alterations in TGF- $\beta$ and $W n t / \beta$-catenin signaling pathways in end organs. In addition, the variability in exosome concentration in end organ tissue according to adipose mass (i.e., dose effect) will need to be clarified. Also, because we did not profile miRNA expression in the adipocytes themselves, we cannot ascertain whether exosomal miRNA differences are a function of different intracellular miRNA abundance or selective miRNA export.

In summary, there are several important findings from this study. First, the bead-based flow cytometry techniques we used to quantify exosomes are generalizable to many laboratories. Second, we show that visceral adipose sheds exosomes primarily of adipocyte, not macrophage, origin. Finally, these exosomes contain miRNAs capable of regulating end-organ TGF- $\beta$ and Wnt $/ \beta$-catenin signaling in obesity-related comorbid conditions. Because these same pathways are important in development, childhood obesity may result in early and cumulative epigenetic changes leading to obesity-related comorbid diseases.

\section{METHODS}

\section{Study Participants}

Participants were females, 11-19 y old. Obese subjects were recruited from our adolescent bariatric surgery program and lean subjects were selected from a convenience sample of patients undergoing unrelated abdominal procedures. The clinical criterion for obesity was a BMI $>30 \mathrm{~kg} / \mathrm{m}^{2}$ and for lean was a BMI $<25 \mathrm{~kg} / \mathrm{m}^{2}$. Obese participants underwent a 2-wk protein-sparing modified fast in the immediate period prior to surgery. All patients (both lean and obese) were fasted $8 \mathrm{~h}$ prior to surgery. Patients were not on any noteworthy medications. BMI was calculated from height and weight measurements taken on day of surgery for all patients. Visceral adipose was excised from the omentum and subcutaneous adipose from the anterior abdominal wall incision site. All clinical investigations were Institutional Review Board approved. Informed consent was obtained from participants or parental guardians. The study enrolled only female participants with age-matched lean controls in an effort to eliminate confounding effects due to sex differences, as in previously published studies (35).

\section{Exosome Isolation}

Visceral and subcutaneous adipose samples collected from obese and lean patients intraoperatively were promptly cultured using a published protocol (12). To isolate exosomes, visceral and subcutaneous adipose tissue were washed with PBS and cut into $4 \mathrm{~mm}^{3}$ pieces, transferred to 12 -well plates containing $3 \mathrm{ml} /$ well of Dulbecco's modified Eagles medium (Invitrogen, Carlsbad, CA) supplemented with $50 \mu \mathrm{g} / \mathrm{ml}$ gentamicin. The culture supernatant was centrifuged at $3,000 \mathrm{~g}$ for $15 \mathrm{~min}$ to remove cells and cellular debris. Exosomes 
were then isolated from cultured supernatants using ExoQuick-TC Precipitation Solution (System Biosciences, Mountain View, CA) and filtered through a $200 \mathrm{~nm}$ filter (Sarstedt, Nümbrecht, Germany).

\section{Nanoparticle Tracking}

The sizes and zeta potentials of the exosomes were determined using a Zetasizer Nano ZS (Malvern Instruments, Worcestershire, UK). Sizing of exosomes was performed using a $10 \mu \mathrm{g} / \mathrm{ml}$ suspension of the exosomes in Milli-Q water (Millipore, Billerica, MA). Zeta potential of the exosomes was measured in Milli-Q water (Millipore) using the Smoluchowski diffusion equation.

\section{Flow Cytometry}

A novel bead-based flow cytometry quantification assay was adapted from Lässer et al. (21). Isolated adipocyte-derived exosomes and exosomal standards (HansaBiomed, Tallinn, Estonia) (0.6, 3, 4.5, 6, 10, and $12 \mu \mathrm{g}$ concentrations) were labeled with PKH67 Green Fluorescent Cell Linker Kit for General Cell Membrane Labeling (Sigma Aldrich, St Louis, MO) using exosome-depleted Fetal Bovine Serum (Lonza, Walkersville, MD (36)) and bound to latex beads (Invitrogen, Carlsbad, CA) coated with anti-CD63 clone H5C6 (BD Biosciences, San Jose, CA). Data were acquired using a FACSCaliber flow cytometer and analyzed using FlowJo (TreeStar, Ashland, OR).

Adipocyte-derived exosomes were characterized using the Lässer et al. protocol for characterizing exosomes (21). Bead-bound exosomes were characterized using the following antibodies: Polyclonal Rabbit Anti-FABP4 (Bioss, Freiburg, Germany), Anti-CD14-PE Clone M5E2 (BD Biosciences), and Anti-DLK/Pref-1-FITC Clone 24-11 (MBL International, Woburn, MA) bound to Goat Anti-Rabbit IgG-Alexa Fluor 700 (Life Technologies, Carlsbad, CA) with appropriate isotype controls: PE Mouse IgG2ak Clone G155-178 (BD Biosciences), Rabbit Anti-IgG (antibodies-online.com, Atlanta, GA), and Rat AntiIgG1-FITC Clone LO-DNP-1 (MBL International). We also stained for endothelial marker Anti-CD31-Alexa 488 Clone M89D3 (BD Biosciences) with appropriate isotype control: Mouse $\operatorname{IgG}_{2 \mathrm{a}}, \kappa$ Alexa Fluor 488 Clone G155-178 (BD Biosciences).

\section{Immunohistochemistry and Immunofluorescence}

Visceral and subcutaneous adipose were stained for M1 and M2 macrophages since adipose macrophage activation state (proinflammatory, or M1, vs. anti-inflammatory, or M2) has been shown to play a role in obesity mediated mechanisms in previous studies (17). Flash frozen visceral and subcutaneous adipose samples were cut into 5 $\mu \mathrm{m}$ sections (Histoserv, Germantown, MD) for M1:M2 Macrophage immunohistochemistry staining using a previously established protocol (17). Antibodies targeting M1 and M2 macrophages subtypes, respectively, were used: CD40 Clone 5C3 (BD Biosciences), CD206 Clone 19.2 (BD Biosciences), and CD163 Clone EDHu-1 (Abdserotec, Oxford, UK). Stained cells were counted in three sections per condition and adipose depot at $20 \times$ magnification and the mean was then calculated.

A549 cells were applied (6,500 cells/well) to eight-well Millicell EZ slides using standardized protocols (37). Cultures were proliferated in standard media (Ham's F12, 10\% FBS, and PenStrep) to $80 \%$ confluence over $24 \mathrm{~h}$. We labeled obese visceral adipocyte exosomes with $\mathrm{PKH} 26$ for $30 \mathrm{~min}$ followed by washes and resuspension at a final concentration of $2 \mu \mathrm{g} / \mathrm{ml}$. We exposed the A549 cells to the PKH26labeled exosomes for $24 \mathrm{~h}$. We then fixed the slides using $4 \%$ paraformaldehyde and added DAPI nuclear stain for viewing and imaging. The chamber slides and exosomes were visualized with an Olympus BX61 upright bright field/fluorescent imaging microscope (Olympus, Center Valley, PA).

\section{RNA Extraction and Amplification}

We extracted adipocyte-exosomal total RNA using mirVana miRNA Isolation Kits (Life Technologies) and amplified total RNA with the Complete Seramir Exosome RNA Amplification Kit from Media and Urine (System Biosciences, Mountain View, CA) according to manufacturer's instructions. A549 cellular RNA was isolated and purified using the Norgen RNA Clean-Up and Concentration Micro Kit (Norgen Biotek, Thorold, Ontario, Canada). We verified amplified exosomal RNA using $1.5 \%$ agarose gel electrophoresis according to manufacturer's instructions and using appropriate validated endogenous controls for qRT-PCR (u6snRNA). We accepted samples of RNA quality determined by Nanodrop1000 (Thermo Scientific, Wilmington, DE) with absorbance ratios for UV $260 / 280 \geq 2.0$ and 260/230 between 1.8 and 2.2.

\section{RNA Expression, Statistical Analyses, and Biological Pathway \\ Analysis}

We used the Agilent 2100 Bioanalyzer RNA Pico Chip (Agilent Technologies, Santa Clara, CA) and TaqMan Gene Expression Assays (Applied Biosystems, Foster City, CA) for detection of GAPDH mRNA. We used TaqMan Small RNA Assays (Invitrogen, Life Technologies) to verify u6snRNA expression. We labeled RNA with Affymetrix FlashTag Biotin HSR RNA Labeling Kit (Affymetrix, Santa Clara, CA) and ran according to standard procedures. Labeled RNA was hybridized to Affymetrix GeneChip miRNA 3.0 arrays which were run using the Fluidics Station 450 Protocol (FS450_002) (Affymetrix, Santa Clara, CA). Resulting data were analyzed in Expression Console using RMA+DMBG (Affymetrix) and then exported it to Partek Genomics Suite for analyses. Only mature human miRNAs $(N=$ 1,733 ) were retained for statistical comparison between groups (as the Affymetrix 3.0 miRNA arrays contain miRNA from multiple species). Three-factor ANCOVA (ID ${ }^{\star}$ group (lean vs. obese)*adipose depot; with age covariate) was used to compare miRNA expression, using unadjusted $P \leq 0.05$ and fold change $\geq|1.2|$ as filters. Main effects for group (obesity-state) and tissue depot were generated along with their interaction term. As a part of the overall ANCOVA, specific withingroup (depot effects within obese or lean) and within-tissue (obesity effects within depot) contrasts were used. Given the relatively small number of comparisons for array analyses, we did not use a False Discovery Rate cutoff, as the purpose of this initial scan was to generate a robust list for pathway analysis (in which type I or false positive results are minimized given the improbability of finding related elements as independent errors). Instead, we used lower cutoffs within downstream pathway analyses.

In Ingenuity Pathways Analysis software (Ingenuity), predicted targets were generated from significantly dysregulated miRNAs. First, significant miRNAs were uploaded from ANCOVA into Ingenuity Pathways Analysis software (Ingenuity). These were processed by Ingenuity's miRNA Target Filter analysis tool, generating target mRNA lists from only experimentally verified or highly conservative predicted targets (a stringent cutoff). Resultant mRNA lists were analyzed for the representation of common biological pathways using Ingenuity's core analysis function and a stringent cutoff for pathway representation at $P \leq 0.01$.

Data discussed in this publication have been deposited in NCBI's Gene Expression Omnibus and are accessible through GEO Series accession numbers GSE50574 (http://www.ncbi.nlm.nih.gov/geo/ query/acc.cgi?acc=GSE50574), and GSE54606 (http://www.ncbi. nlm.nih.gov/geo/query/acc.cgi? token $=$ mpqlimievtsfbob\&acc $=$ GSE54606).

\section{qRT-PCR}

The Individual MicroRNA Assays (Life Technologies) used for confirmation were: miR-23b, miR-148b, miR-4269, and miR-4429. Specifications for the TaqMan MicroRNA Assays include: TaqMan MicroRNA Reverse Transcription Kit (Life Technologies), TaqMan PreAmp Master Mix (2X) (Life Technologies), and the TaqMan Universal Master Mix II, No AmpErase UNG (2X) (Life Technologies).

A549 cellular RNA was processed with the High Capacity cDNA Reverse Transcription Kit (Life Technologies), TaqMan Gene Expression Master Mix (Life Technologies), and the TaqMan Gene Expression Assays for ACVR2B and GAPDH (endogenous control) (Life Technologies). All miRNA and mRNA results were analyzed with SDS (Applied 7900HT Fast Real-Time PCR System, Life Technologies) and Microsoft Excel 2013 (Microsoft, Redmond, WA).

\section{Data and Materials Availability}

The data discussed in this publication have been deposited in NCBI's Gene Expression Omnibus (38) and are accessible through GEO Series accession number GSE50574 (http://www.ncbi.nlm.nih.gov/ geo/query/acc.cgi?acc=GSE50574). 


\section{SUPPLEMENTARY MATERIAL}

Supplementary material is linked to the online version of the paper at http:// www.nature.com/pr

\section{STATEMENT OF FINANCIAL SUPPORT}

This project was supported by grants R01MD007075 and UL1TR000075 to R.J.F. and by grant K12HD001399 to D.K.P. from the National Institutes of Health, Bethesda, MD. Additional support was provided to R.J.F. by the Clark Charitable Foundation, Bethesda, MD.

Disclosure: There is no conflict of interest to disclose.

\section{REFERENCES}

1. Xu H, Barnes GT, Yang Q, et al. Chronic inflammation in fat plays a crucial role in the development of obesity-related insulin resistance. J Clin Invest 2003;112:1821-30.

2. Lumeng CN, Saltiel AR. Inflammatory links between obesity and metabolic disease. J Clin Invest 2011;121:2111-7.

3. Jensen ME, Gibson PG, Collins CE, Wood LG. Airway and systemic inflammation in obese children with asthma. Eur Respir J 2013;42: 1012-9.

4. de Jong PE, Verhave JC, Pinto-Sietsma SJ, Hillege HL; PREVEND study group. Obesity and target organ damage: the kidney. Int J Obes Relat Metab Disord 2002;26:Suppl 4:S21-4.

5. Fontana L, Eagon JC, Trujillo ME, Scherer PE, Klein S. Visceral fat adipokine secretion is associated with systemic inflammation in obese humans. Diabetes 2007;56:1010-3.

6. Fain JN, Tichansky DS, Madan AK. Transforming growth factor beta1 release by human adipose tissue is enhanced in obesity. Metab Clin Exp 2005;54:1546-51.

7. Masszi A, Fan L, Rosivall L, et al. Integrity of cell-cell contacts is a critical regulator of TGF-beta 1-induced epithelial-to-myofibroblast transition: role for beta-catenin. Am J Pathol 2004;165:1955-67.

8. Akhmetshina A, Palumbo K, Dees C, et al. Activation of canonical Wnt signalling is required for TGF- $\beta$-mediated fibrosis. Nat Commun 2012;3:735.

9. Fujii H, Kawada N. Inflammation and fibrogenesis in steatohepatitis. J Gastroenterol 2012;47:215-25.

10. Camussi G, Deregibus MC, Bruno S, Grange C, Fonsato V, Tetta C. Exosome/microvesicle-mediated epigenetic reprogramming of cells. Am J Cancer Res 2011;1:98-110.

11. Zernecke A, Bidzhekov K, Noels H, et al. Delivery of microRNA-126 by apoptotic bodies induces CXCL12-dependent vascular protection. Sci Signal 2009;2:ra81.

12. Deng ZB, Poliakov A, Hardy RW, et al. Adipose tissue exosome-like vesicles mediate activation of macrophage-induced insulin resistance. Diabetes 2009;58:2498-505.

13. Ardoin SP, Shanahan JC, Pisetsky DS. The role of microparticles in inflammation and thrombosis. Scand J Immunol 2007;66:159-65.

14. Lässer C, Alikhani VS, Ekström K, et al. Human saliva, plasma and breast milk exosomes contain RNA: uptake by macrophages. J Transl Med 2011;9:9.

15. Camussi G, Deregibus MC, Bruno S, Cantaluppi V, Biancone L. Exosomes/ microvesicles as a mechanism of cell-to-cell communication. Kidney Int 2010;78:838-48.

16. Kishida K, Funahashi T, Matsuzawa Y, Shimomura I. Visceral adiposity as a target for the management of the metabolic syndrome. Ann Med 2012;44:233-41.
17. Aron-Wisnewsky J, Tordjman J, Poitou C, et al. Human adipose tissue macrophages: $\mathrm{m} 1$ and $\mathrm{m} 2$ cell surface markers in subcutaneous and omental depots and after weight loss. J Clin Endocrinol Metab 2009;94:4619-23.

18. Imaizumi K, Kawabe T, Ichiyama S, et al. Enhancement of tumoricidal activity of alveolar macrophages via CD40-CD40 ligand interaction. Am J Physiol 1999;277(1 Pt 1):L49-57.

19. Fujisaka S, Usui I, Bukhari A, et al. Regulatory mechanisms for adipose tissue M1 and M2 macrophages in diet-induced obese mice. Diabetes 2009;58:2574-82.

20. Mathivanan S, Ji H, Simpson RJ. Exosomes: extracellular organelles important in intercellular communication. J Proteomics 2010;73:1907-20.

21. Lässer C, Eldh M, Lötvall J. Isolation and characterization of RNA-containing exosomes. J Vis Exp 2012;59:e3037.

22. Février B, Raposo G. Exosomes: endosomal-derived vesicles shipping extracellular messages. Curr Opin Cell Biol 2004;16:415-21.

23. Shan T, Liu W, Kuang S. Fatty acid binding protein 4 expression marks a population of adipocyte progenitors in white and brown adipose tissues. FASEB J 2013;27:277-87.

24. Wang Y, Kim KA, Kim JH, Sul HS. Pref-1, a preadipocyte secreted factor that inhibits adipogenesis. J Nutr 2006;136:2953-6.

25. Khazen W, M'bika JP, Tomkiewicz C, et al. Expression of macrophage-selective markers in human and rodent adipocytes. FEBS Lett 2005;579:5631-4.

26. Kozomara A, Griffiths-Jones S. miRBase: integrating microRNA annotation and deep-sequencing data. Nucleic Acids Res 2011;39(Database issue):D152-7.

27. Santibañez JF, Quintanilla M, Bernabeu C. TGF- $ß / T G F-\beta$ receptor system and its role in physiological and pathological conditions. Clin Sci 2011;121:233-51.

28. Henderson WR Jr, Chi EY, Ye X, et al. Inhibition of Wnt/beta-catenin/ CREB binding protein (CBP) signaling reverses pulmonary fibrosis. Proc Natl Acad Sci USA 2010;107:14309-14.

29. Zavadil J, Böttinger EP. TGF-beta and epithelial-to-mesenchymal transitions. Oncogene 2005;24:5764-74.

30. Hinz B. Formation and function of the myofibroblast during tissue repair J Invest Dermatol 2007;127:526-37.

31. Divoux A, Tordjman J, Lacasa D, et al. Fibrosis in human adipose tissue: composition, distribution, and link with lipid metabolism and fat mass loss. Diabetes 2010;59:2817-25.

32. Spencer M, Yao-Borengasser A, Unal R, et al. Adipose tissue macrophages in insulin-resistant subjects are associated with collagen VI and fibrosis and demonstrate alternative activation. Am J Physiol Endocrinol Metab 2010;299:E1016-27.

33. Lacasa D, Taleb S, Keophiphath M, Miranville A, Clement K. Macrophagesecreted factors impair human adipogenesis: involvement of proinflammatory state in preadipocytes. Endocrinology 2007;148:868-77.

34. Sethi JK. Activatin' human adipose progenitors in obesity. Diabetes 2010;59:2354-7.

35. Buchwald H, Estok R, Fahrbach K, et al. Weight and type 2 diabetes after bariatric surgery: systematic review and meta-analysis. Am J Med 2009;122:248-56.

36. Xiang X, Poliakov A, Liu C, et al. Induction of myeloid-derived suppressor cells by tumor exosomes. Int J Cancer 2009;124:2621-33.

37. Freishtat RJ, Watson AM, Benton AS, et al. Asthmatic airway epithelium is intrinsically inflammatory and mitotically dyssynchronous. Am J Respir Cell Mol Biol 2011;44:863-9.

38. Edgar R, Domrachev M, Lash AE. Gene Expression Omnibus: NCBI gene expression and hybridization array data repository. Nucleic Acids Res. 2002;30:207-210. 